\title{
Fuentes, archivos y debates sobre el movimiento sindical y los trabajadores(as) en Chile durante la dictadura de Pinochet 1973-1990
}

\author{
Rodrigo Araya Gómez \\ Escuela de Historia, Universidad Academia de Humanismo Cristiano, Chile \\ raraya@academia.cl
}

\begin{abstract}
:
The article aims to analyze the relationship between access to sources and archives and the evolution of debates about the role of the trade union movement in the dictatorship Chile. For this, a brief state of the question about labor historiography, its debates and inflection points will be made; later, the main sources on the history of the trade union movement will be identified in the dictatorship, to raise the potential and challenges of its examination, which can contribute to the renewal of studies and to a more complete and documented reconstruction of this crucial period in Chile's recent history.
\end{abstract}

KEYWORDs: Trade unionism, Dictatorship, Neoliberalism, Historiography.

\section{ReSUMEN:}

El artículo tiene por objetivo analizar la relación existente entre el acceso a las fuentes y archivos y la evolución en los debates sobre el rol del movimiento sindical en el Chile de la dictadura. Para ello, se realizará un breve estado de la cuestión sobre la historiografía laboral, sus debates y puntos de inflexión; posteriormente se identificarán las principales fuentes sobre historia del movimiento sindical en dictadura, para plantear la potencialidad y desafíos de su examen, que puede contribuir a la renovación de los estudios y a una reconstrucción más acabada y documentada de este crucial periodo de la historia reciente de Chile.

Palabras clave: Sindicalismo, Dictadura, Neoliberalismo, Historiografía.

\section{INTRODUCCIÓN}

El artículo tiene por objetivo analizar la relación existente entre el acceso a las fuentes y archivos y la evolución en los debates sobre el rol del movimiento sindical en el Chile de la dictadura. Para ello realizaremos una breve síntesis de la evolución de la historiografía del movimiento sindical chileno, de manera de comprender el impacto del golpe de Estado del 11 de septiembre de 1973 como un hito o punto de quiebre para el desarrollo de los estudios sobre el mundo del trabajo y la historiografía de carácter marxista. En este sentido, en un primer apartado procederemos a analizar la evolución de los estudios sindicales durante la dictadura, destacando los aportes de algunos de sus principales representantes en la década de los 80 hasta la situación actual de las investigaciones históricas sobre el actor sindical. Posteriormente, en el segundo apartado analizaremos los tipos de fuentes y archivos existentes para estudiar el movimiento sindical y sus posibilidades para profundizar en el conocimiento de la dictadura cívicomilitar.

La valoración de las fuentes existentes y su potencialidad para estudiar el movimiento sindical es relevante dada la escasez de estudios históricos sobre el movimiento sindical durante la dictadura cívico-militar. Esto resulta contrastante con el énfasis puesto en las investigaciones dedicadas al estudio de otros movimientos sociales, o del sindicalismo, por otras áreas de las ciencias sociales, especialmente la sociología. Así, sostenemos la existencia de una relación directa entre el desarrollo de la historiografía del movimiento sindical y las variaciones en la trayectoria del actor sindical como protagonista del proceso político chileno. De este modo, la pérdida de relevancia del movimiento sindical durante la dictadura, y especialmente durante los gobiernos 
democráticos, habría redundado en una falta de interés por las investigaciones sobre el sindicalismo, que acaba por ser considerado un apéndice marginal en los estudios relativos al mundo del trabajo.

En este sentido, planteamos que la historia del movimiento sindical ha experimentado un proceso de renovación en los últimos años, debido al desarrollo de nuevos tipos de acción sindical, protagonizados por trabajadores pertenecientes a algunas de las áreas más dinámicas de la economía chilena, como la minería, la pesca, industria forestal y el retail, entre otras ${ }^{1}$. Este desarrollo se ha visto estimulado por la confluencia de intereses de investigadores de otras ramas de las ciencias sociales, preocupados también por el análisis de los problemas y conflictos del mundo del trabajo. Esta preocupación viene a confirmar la actualidad del concepto de trabajo como elemento clave para entender las dinámicas que experimenta la sociedad chilena luego de décadas de aplicación del modelo neoliberal (Ruiz, 2015), (Garretón, 2012), (Gaudichaud, 2017). De este modo, el reimpulso en los estudios sobre el movimiento sindical ha significado también el uso de nuevas fuentes y archivos, hecho que además ha generado distintos enfoques metodológicos que son consecuencia de la interdisciplinariedad de muchas de las investigaciones.

Por lo tanto, existiría una directa relación entre el desarrollo del proceso político, el surgimiento de los partidos de izquierda y la historiografía laboral. Ahora bien, a pesar de su importancia en la historia social chilena, el movimiento sindical no ha sido objeto preferente de estudio para la historiografía. En efecto, los trabajos sobre temáticas vinculadas al desarrollo del sindicalismo surgieron a mediados del siglo XX con la emergencia en paralelo de la historiografía de carácter marxista (Jobet, 1951) (Ramírez Necochea, 1956), (Ortiz Letelier, 1956) ${ }^{2}$.

En la década siguiente, y hasta 1973, alcanzará su auge la historiografía marxista, mientras que los estudios laborales se verán enriquecidos con el aporte de la sociología del trabajo. Esta disciplina, mediante la combinación de estudios de casos y la realización de entrevistas, analizará las características del movimiento sindical chileno. Esas investigaciones se profundizarán al estudiar in situ la experiencia del gobierno de la Unidad Popular, encabezada por el presidente socialista Salvador Allende. En este mismo periodo, el movimiento sindical chileno alcanzó un alto grado de madurez político-social expresada en la acción de la Central Única de Trabajadores (CUT), vigente entre 1953 y 1973 (Samaniego, 2016). La CUT, organización heredera de las históricas organizaciones $\mathrm{FOCH}$ y $\mathrm{CTCH}$, adhirió a un proyecto global de transformación de la sociedad. Este hecho se expresa en su apoyo al proyecto socialista encarnado en un primer momento por el Frente de Acción Popular y posteriormente por la Unidad Popular, que vincula su destino al proyecto socialista liderado por el presidente Salvador Allende. De este modo, el movimiento sindical en su gran mayoría apoyó a la Unidad Popular, y se convirtió en un reflejo de los principales conflictos del periodo. Esas tensiones terminaron debilitando al sindicalismo en su conjunto, que quedó en situación de crisis. En esos momentos se producirá el golpe de Estado del 11 de septiembre de 1973, hito clave para comprender la trayectoria del sindicalismo de los últimos 40 años.

Ahora bien, junto con este quiebre democrático o fin de una trayectoria política ininterrumpida desde 1932, esta primera etapa de los estudios del mundo del trabajo, con fuerte impronta marxista y estructuralista, sufre un abrupto quiebre con el golpe de Estado del 11 de septiembre de 1973, que se expresa en una ruptura "histórica" y "epistemológica" (Salazar, 1985). Además, los principales representantes de la historiografía fueron al exilio o incluso se convirtieron en detenidos desaparecidos -como fue el caso de Ortiz Letelier-, mientras que unidades académicas vinculadas a las ciencias sociales son suprimidas, de manera que este quiebre si sintió tanto en el plano personal como en el ámbito de la academia.

\section{DE LA NUEVA HISTORIA SOCIAL A LA TEORÍA DE LOS MOVIMIENTOS SOCIALES}

Como hemos señalado, el golpe de Estado implicó una ruptura para el desarrollo de la historiografía marxista chilena y, como efecto de ello, un quiebre también para la historia social. Comprendido el golpe como un hito refundacional -con los principales representantes de la historia del movimiento sindical en 
estado de dispersión o persecución-, los estudios sobre el mundo del trabajo tuvieron escasa relevancia a nivel de las universidades chilenas. Deben exceptuarse, sin embargo, los trabajos de DERTO ${ }^{3}$ y el de José Isla y otros autores sobre estadísticas sindicales ${ }^{4}$, los cuales sirvieron para medir el grado de evolución de la afiliación sindical. Esos datos fueron especialmente útiles para evaluar el impacto de la represión dictatorial en las tasas de sindicalización, y, a la vez, para graficar el aumento significativo de la afiliación sindical durante el periodo final del gobierno de Frei Montalva y la Unidad Popular.

Desde el primer momento, la dictadura cívico-militar aplicó una dura política represiva contra el movimiento sindical, fundamentada tanto en el histórico vínculo entre la izquierda y el movimiento obrero (Garcés y Milos, 1988), como también en el importante rol que había desempeñado la patronal en la gestación del golpe de Estado y en la posterior afirmación del bloque en el poder (Campero, 1984). Esta participación le permitió apoyar y colaborar con la represión de las dirigencias sindicales para asegurar la conformación de un actor sindical pasivo o complaciente con las nuevas autoridades en particular, y en general con el nuevo orden político autoritario (Bohoslavsky, Fernández y Smart, 2019).

Sin embargo, junto a la represión, los efectos más devastadores de la dictadura se generaron a partir de la aplicación del modelo neoliberal por parte de las autoridades militares y sus colaboradores civiles, pertenecientes al equipo de economistas conocidos como "Chicago boys" (Gárate, 2012). En efecto, el cambio de modelo económico significó el deterioro de las condiciones de vida de los trabajadores y sus familias, y, junto a ello, un fuerte proceso de desindustrialización que afectó fundamentalmente a las áreas económicas de donde el sindicalismo extraía su fuerza, como la del metal, la minera y la textil. En 1979 se impuso una nueva normativa, el Plan Laboral, el cual se convirtió en el otro hito o punto de quiebre en la trayectoria del sindicalismo chileno. Así, este conjunto de normas buscó la liberalización de las relaciones laborales y el debilitamiento y despolitización de los sindicatos, de manera de adaptar el mundo del trabajo al nuevo modelo de economía abierta.

Entonces, la magnitud de los cambios experimentados por la clase trabajadora sirvió de estímulo a un grupo de sociólogos vinculados a organizaciones no gubernamentales para estudiar el impacto del Plan Laboral en el sindicalismo. Así, desde el área de la sociología se estudiaron, a fines de la década de 1970 y comienzos de la década de 1980, los efectos del Plan Laboral en el mundo del trabajo y las respuestas sindicales a las políticas laborales de la dictadura. De ello surgen textos de referencia como los de Campero y Valenzuela (1981); Barrera, Henríquez y Selame (1985); Ruiz-Tagle (1985), y Frías (1988).

En general estos trabajos respondieron a enfoques basados en el estructuralismo y se plantearon visualizar posibles escenarios de acción sindical en un contexto marcado por grandes transformaciones en la estructura social chilena.

Guillermo Campero y José Antonio Valenzuela realizaron una de las primeras y más detalladas investigaciones sobre el impacto de la dictadura en el movimiento sindical. Estos autores describieron -con base en estadísticas oficiales y documentos emanados de la Vicaría de la Solidaridad, Vicaría de la Pastoral Obrera y diversos artículos publicados por revistas pertenecientes a ONG’s de la época- la evolución del sindicalismo en el periodo, combinada con las variaciones de la actividad económica y sus efectos en las organizaciones sindicales. Junto a ello, analizaron las relaciones desarrolladas entre personeros de la dictadura y dirigentes sindicales vinculados a la democracia cristiana y el gremialismo. De este modo, Campero y Valenzuela establecieron escenarios y orientaciones para la acción sindical en una nueva fase de la dictadura post Plan Laboral, que abre la posibilidad de una recuperación del movimiento sindical.

Jaime Ruiz-Tagle, en un libro compilatorio de trabajos anteriores ${ }^{5}$, analizó los efectos del Plan Laboral en el sindicalismo, y destacó la posibilidad de conformación de un nuevo movimiento sindical producto de la reactivación de la acción sindical a nivel de empresa. Además, según el autor, el nuevo contexto laboral favorecería la autonomía del sindicalismo y el desarrollo de nuevos valores vinculados a la recuperación de la democracia. Es decir, el sindicalismo buscaría reconstituirse como un actor nacional para así desempeñar un rol relevante en la nueva democracia que se buscaba reconquistar. 
Manuel Barrera, Helia Henríquez y Teresa Salamé investigaron los efectos de la puesta en vigor de la nueva modalidad de negociación colectiva a partir del dictado del Plan Laboral. Para ello, los autores recurrieron a una metodología de tipo participativa para conocer las opiniones de los dirigentes sindicales, profesionales vinculados a los sindicatos e investigadores sindicales. Las conclusiones a las que llegaron coincidieron en general con los planteamientos de Ruiz-Tagle. Respecto a la reanudación de la negociación colectiva, la misma habría servido de estímulo para una tímida reactivación del movimiento sindical, el cual criticó fuertemente el bajo porcentaje de reajuste de los salarios, valoró las posibilidades de fortalecimiento interno de los sindicatos, y la recuperación de su unidad en un contexto marcado por el miedo a la cesantía y a la represión.

Patricio Frías - destacado sociólogo, doctorado en la Universidad de Lovaina-, por su parte, realizó una serie de investigaciones en la década de los 80 , basadas en una multiplicidad de fuentes provenientes de documentos internos de organizaciones sindicales, estadísticas y noticias de prensa. En ellas planteó que el sindicalismo desarrolló lógicas de acción de acuerdo a la coyuntura y a la correlación de fuerzas respectiva, y estableció una línea de interpretación que continuará en sus trabajos posteriores a la década de los 90 .

Si bien estas investigaciones corresponden a trabajos tributarios de un contexto marcado por la imposición del modelo neoliberal por parte de la dictadura de Pinochet y la aplicación del Plan Laboral, aquéllas tienen un valor referencial para la mayoría de las obras generales sobre la dictadura, pues se refieren en forma tangencial al sindicalismo, al citar precisamente estos estudios provenientes de la sociología del trabajo.

El predominio de los enfoques sociológicos sobre el movimiento sindical durante la dictadura cívicomilitar da cuenta de la escasez de trabajos historiográficos sobre el tema. Este "vacío historiográfico" se explicaría por la pérdida del trabajo como categoría analítica, y del movimiento sindical como actor social relevante. En este sentido, si consideramos los balances historiográficos realizados por Jorge Rojas Flores (2000) y Ángela Vergara (2018), aquéllos dan cuenta del predominio de corrientes historiográficas culturalistas o vinculadas a la historia social, en los que una de las premisas fue el retorno del sujeto y la reconstrucción de los contextos como factores constitutivos de la identidad de los individuos.

Así, la historiografía chilena experimentó un proceso de renovación, conformado principalmente por los llamados representantes de la Nueva Historia Social desarrollada en el exilio y con influencias posteriores en Chile (Salazar, 1985; Grez, 1997; Pinto, 1998; Garcés y De la Maza, 1985). Estos autores se dedicaron a estudiar los sectores populares y los procesos de surgimiento del proletariado, que habían sido abandonados por los representantes de la historiografía marxista clásica. De este modo, estos trabajos vinieron a diversificar el estudio de los sujetos subalternos en sus experiencias cotidianas y procesos de politización temprana, énfasis que según este grupo de historiadores había sido descuidado por los representantes de la historiografía marxista, más preocupada - paradójicamente- por estudiar los sistemas de dominación, lo que reproducía la invisibilidad estructural de los sectores populares.

Por lo tanto, esta opción epistemológica implicó una baja producción historiográfica relativa al estudio del movimiento sindical durante la dictadura, salvo excepciones como la clásica obra de Crisóstomo Pizarro sobre la huelga general (1985).

No obstante, en los últimos años se ha producido una renovación del interés por los estudios sobre historia del sindicalismo, que incluye al estudio de la acción sindical durante la dictadura. Así, tenemos los trabajos de Álvarez (2010 y 2011), López (2012), Araya (2012, 2014, 2015, 2017). En el caso del primero, el análisis se centra en el estudio de las políticas laborales ejecutadas por la dictadura, las cuales oscilan entre intentos de cooptación y de represión directa encaminada a reducir a un rol mínimo al sindicalismo como actor social. En el trabajo de López, el énfasis está colocado en la resistencia ejercida por las organizaciones sindicales en contra de la dictadura en el periodo más intenso de la represión. En el caso de Araya, el interés es reconstruir la trayectoria del movimiento sindical a partir de la acción de las organizaciones sindicales de carácter nacional, de manera tal de rescatar el aporte del sindicalismo en la lucha por la recuperación de la democracia.

Además, cabe destacar la producción historiográfica originada en el extranjero, desde el trabajo pionero de Alan Angell (1974), a los aportes de Joel Stillerman (2005), Paul Drake (1993 y 1996), Thomas Klubock 
(2012), Peter Winn (2005 y 2012), Heidi Tinsmann (2017) y Volker Frank (2000, 2012), entre otros, quienes han estudiado los efectos del Plan Laboral en el movimiento sindical, y han coincidido en la situación de crisis del sindicalismo y en su reactivación durante la década de los 80 , a pesar de la cual los sindicatos no alcanzaron a recuperar el poder que habían tenido anteriormente.

Ahora bien, como se ha señalado anteriormente, la mayoría de los primeros estudios sobre el movimiento sindical en la dictadura tuvieron un carácter sociológico, los cuales hasta la actualidad han constituido las obras de referencia para los trabajos generales sobre la dictadura y los gobiernos democráticos; textos que tienen como objetivo identificar los efectos del Plan Laboral en el modelo de relaciones laborales vigente. Esta misma referencia a la continuidad del legado de la dictadura ha sido empleada para justificar los constantes debates o discusiones sobre los proyectos de reforma laboral, los cuales han sido propuestos de manera constante desde el año 1990 en adelante, problemática que nos lleva a preguntarnos sobre los grados de legitimidad del modelo laboral.

Esta evaluación del legado de la dictadura en el plano laboral se ha hecho presente en los últimos años, a propósito de investigaciones que se han centrado en el movimiento de trabajadores subcontratistas en diferentes ramas de la actividad económica. Aquí se destacan los estudios de Aravena y Núñez (2009), Zapata (2010), además de otros trabajos que dan cuenta de una renovación de los estudios sobre el trabajo -Osorio (2015), Ayala (2016), Pinto, Ponce y Santibáñez (2017)-.. Estos trabajos en general han combinado el trabajo interdisciplinario entre la historia y la sociología, empleando conceptos tributarios de la teoría de los movimientos sociales, que han servido para analizar la acción sindical en el Chile de la sociedad de la posdictadura.

Además, han surgido iniciativas académicas que han tendido a analizar problemas relativos a la precariedad, flexibilización y tercerización de las relaciones laborales, entre las que se destacan el aporte del Grupo de Estudios del Trabajo desde el Sur (GETSUR), la Fundación Sol, Centro de Investigación Político Social del Trabajo (CIPSTRA), y el Grupo de estudios de acción colectiva, trabajo y conflictos sociales de la Facultad de Ciencias Sociales de la Universidad de Chile.

Ahora bien, la escasez de trabajos sobre el sindicalismo en la dictadura nos lleva también a preguntarnos sobre las fuentes y archivos que contienen documentación sobre ellos; en otras palabras, interrogarnos sobre la viabilidad de las investigaciones sobre el tema, problema que veremos en el siguiente apartado.

\section{DE FUENTES, ARCHIVOS Y DOCUMENTOS. El MUNDO DEL TRABAJO A TRAVÉS DE SUS HUELLAS.}

Los estudios sobre el mundo del trabajo se han visto dificultados por una serie de factores:

- la dispersión de las fuentes y archivos.

- la ausencia de una política archivística por parte de las organizaciones sindicales.

- la falta de preocupación del Estado respecto al resguardo de las memorias de los movimientos sociales.

En el primer caso, se puede constatar como problema la dispersión de las fuentes provenientes de organizaciones sindicales, situación que también comprendería a las antiguas centrales nacionales, como la FOCH y la CTCH. Este problema respondería a una característica histórica del movimiento sindical chileno, su debilidad organizacional, traducida en la falta de consolidación de sus instancias intermedias de organización, como federaciones de rama o sindicatos provinciales. Problema que se repetiría con mayor frecuencia en la mediana y pequeña empresa, en las que el sindicalismo tenía poca presencia.

Por lo tanto, las organizaciones sindicales no produjeron documentación interna en gran cantidad, o tuvieron dificultades en conservarla, por los distintos periodos en que sufrieron la represión por parte del Estado. Estas acciones en muchos casos implicaron destrucción o confiscación de documentación interna. Este problema se hizo aún más evidente con la política antisindical de la dictadura, puesto que numerosas organizaciones o bien se ilegalizaron, como la CUT, la Federación Sindical del Metal o la Confederación 
Ranquil, o bien se dificultó de tal manera su accionar que terminaron por desaparecer, como fue el caso del SUTE, Sindicato Único de Trabajadores de la Educación (SUTE), sin que se tuviera claridad sobre el destino de su documentación. Aquí cabe agregar un problema adicional, puesto que en muchos casos, la documentación sindical requisada pasó a poder de los organismos de seguridad. Así, por pertenecer al área de la Defensa, estos organismos están excluidos - mediante la ley 18.771- de la obligación que tienen los demás organismos públicos de transferir su documentación al Archivo Nacional, lo que obstaculiza el acceso a esa fuente de información ${ }^{6}$. Entonces, algunos documentos de carácter sindical, así como otros originados en el periodo dictatorial, aparecen en trabajos periodísticos, como los de Mauricio Weibel y Jorge Escalante, sin revelarse el origen de las fuentes, lo que da cuenta de la existencia de verdaderas colecciones privadas de documentos históricos.

No obstante, se puede encontrar información de carácter sindical en algunos fondos del Archivo Nacional de la Administración (ARNAD), entre los que se destaca en primer lugar el Fondo de Organizaciones Sociales, originado a partir de la donación realizada por la ONG ECO Comunicaciones. Este fondo posee documentación emanada de sindicales locales, organizaciones intermedias, y de otros organismos que tuvieron algún tipo de relación con el sindicalismo opositor como fue la Vicaría de Pastoral Obrera. La información es relevante sobre todo desde un punto de vista cualitativo, porque da cuenta de las trayectorias, tensiones internas y relaciones con el contexto histórico en que se desenvolvían, además de recoger testimonios de testigos de la época, que permiten reconstruir un cuadro más detallado de la acción sindical en el periodo.

Otros fondos poco explorados corresponden a los de origen estatal, como el del Ministerio del Trabajo, Dirección del Trabajo y Dirección de Relaciones Laborales (DIRELA), todos ellos contenidos en el ARNAD. Ahora bien, en el caso de los dos primeros se presenta el problema de la escasez de volúmenes para el periodo dictatorial, déficit que se puede explicar por la insuficiente cantidad de documentación transferida al ARNAD. En este sentido, si bien el Estado garantiza el libre acceso de los ciudadanos a los archivos, este derecho tiene dificultades para concretarse debido a las diferencias de políticas de resguardo y conservación de la documentación por parte de las instituciones públicas, como ocurre en el caso de la Dirección del Trabajo, institución que sólo tiene parte de su documentación en el ARNAD hasta el año 1985 aproximadamente, mientras que la información restante continúa sin ser transferida. No obstante, los volúmenes que han podido ser revisados presentan valiosa información sobre los problemas cotidianos que enfrentaron algunos sindicatos, el estricto control de los fiscalizadores de la dirección hacia las directivas sindicales, expresado en solicitudes de inhabilidad de dirigentes o la revisión de cuentas de los sindicatos, materias que permiten conocer aspectos poco investigados de la vida sindical.

Este mismo problema se encuentra en el fondo del Ministerio del Trabajo, el cual contiene escasa documentación sobre los años de la dictadura, especialmente la correspondiente a la década de los 80; por ejemplo, hay 17 volúmenes aproximadamente del año 1983, que contienen oficios, providencias, entre otros documentos que dan cuenta de la dinámica interna del ministerio en el periodo dictatorial. Por lo tanto, para poder acceder a la información de dichas instituciones, se debe completar un formulario, vía ley de transparencia, que impide acceder a grandes volúmenes de información, hecho que resulta una condición esencial para la comprensión de grandes procesos.

Con relación al fondo del DIRELA, fondo escasamente trabajado en los estudios historiográficos sobre el movimiento sindical, se encuentran expedientes de sindicatos que reflejan su trayectoria desde su constitución hasta su desaparición, por voluntad de sus socios o del ministerio de la ley, proceso que se dio especialmente entre los años 1979 y 1981. En este último caso, el análisis de la documentación permite conocer el destino de muchos sindicatos una vez aplicado el Plan Laboral, el cual vino a sincerar las cifras de afiliación sindical, que, de acuerdo a estadísticas oficiales, continuaban en niveles parecidos al periodo anterior a 1973. Así, se puede observar a través de casos concretos el nivel de inactividad de los sindicatos, la designación de directivas por parte de las autoridades de la dictadura y el nivel de represión ejercido. Todo 
esto resulta evidente si se comparan las nóminas de socios y de directivas de los sindicatos entre el periodo anterior y posterior al golpe.

También se puede registrar información de carácter sindical en los centros de documentación del Museo de la Memoria, la Fundación de Ayuda Social de las Iglesias Cristianas (FASIC), Londres 38 y la Fundación Vicaría de la Solidaridad. En este caso nos encontramos con ONG's de reconocida trayectoria en la lucha por los derechos humanos como el caso de la Vicaría de la Solidaridad, institución que por más de 15 años luchó por la defensa de los derechos humanos. Esta defensa incluía la promoción de organizaciones sindicales independientes del gobierno -y a la vez claramente identificadas con la oposición-, entre las que se encontraban los grupos sindicales como el Grupo de los Diez y la Coordinadora Nacional Sindical. Este trabajo le permitió a la Vicaría acumular una gran cantidad de información, que puede consultarse en forma presencial en el centro de documentación que resguarda el acervo histórico de la institución. También se puede encontrar información en formato digital en la página web de la institución ${ }^{7}$. En el caso de FASIC, esta institución tiene una larga trayectoria de defensa de los derechos humanos, plasmada tanto en asesorías jurídicas como en la realización de actividades de difusión de información y de protección de las víctimas. Esta institución posee un archivo histórico que contiene información relevante sobre los crímenes de la dictadura y registros de la resistencia en diversos formatos. El acceso a la documentación puede ser presencial o vía web, a través de la página de la institución. En el sitio, además, se pueden visitar las colecciones digitales ${ }^{8}$.

En el caso del Museo de la Memoria y Los Derechos Humanos, y Londres 38, Espacio de Memorias, nos encontramos con instituciones vinculadas a políticas de memoria, que en razón de su actividad tuvieron o tienen contacto con dirigentes sindicales u organizaciones del mismo tenor. Por lo tanto, en razón de su actividad, han recibido documentación que recoge las actividades de la oposición antidictatorial, información a la cual es posible acceder a través de la página web de ambas instituciones - muchos documentos se encuentran allí en formato $\mathrm{PDF}^{9}$-, o en forma presencial. El Museo de la Memoria tiene para ello un importante centro de documentación, el cual incluso posee material donado que todavía se encuentra en proceso de catalogación.

El otro tipo de fuentes donde se puede encontrar información sobre la actividad sindical en la dictadura corresponde a las periódicas, tanto de revistas como periódicos de gobierno y oposición. En este caso, los datos variarán de acuerdo a la línea editorial del medio de prensa o a la coyuntura política, puesto que la censura operará permanentemente en contra de los medios opositores, y se descontinuará la información sobre el sindicalismo antidictatorial. Así, tenemos el diario Fortin Mapocho, surgido como semanario en 1984, y convertido en diario el año 1987; este medio de prensa, vinculado a la oposición contiene diversas noticias sobre la acción de los sindicatos y sirvió de órgano difusor del Comando Nacional de Trabajadores mediante la publicación como inserto del boletín oficial de la organización. Por otro lado, tenemos una serie de revistas de oposición que difundieron los planteamientos de dirigentes sindicales de la oposición, entre las que se destacan Análisis y Hoy, la primera vinculada a la izquierda y la segunda a la democracia cristiana; ambas, a su vez, poseían un amplio abanico de materias que dan cuenta de la complejidad del periodo dictatorial.

Junto a este tipo de fuentes se encuentran las memorias de numerosos dirigentes sindicales, en algunos casos registradas en formato audiovisual, como es el caso de la colección de videos con entrevistas a dirigentes contenida en el Centro de Documentación del Museo de la Memoria. Por lo tanto, observamos las fuentes orales como un complemento de la información contenida en los archivos, además de dar cuenta de la relevancia que adquirió en los últimos años la historia oral como una rama autónoma de la historiografía, en conexión también con la llamada historia reciente o historia del tiempo presente. Esta área todavía se encuentra en un proceso de consolidación, especialmente entre las generaciones más jóvenes de historiadores y en general entre los investigadores pertenecientes a las ciencias sociales.

El segundo factor que ha influido en el desarrollo de los estudios históricos sobre el mundo del trabajo ha sido la ausencia de una política archivística por parte de las organizaciones sindicales. En efecto, las deficiencias en la estructura organizacional de los sindicatos influyeron en la falta de conciencia de la 
necesidad de generar acervos documentales que resguardasen la memoria de las propias organizaciones. Esto, a la vez, impidió que se conocieran sus historias. Igualmente, los efectos perversos del Plan Laboral significaron un debilitamiento económico de los sindicatos, puesto que, al suprimirse la obligación del pago de la cuota sindical, éstos perdieron buena parte de sus fuentes de financiamiento, de modo que la reducción obligada de costos habría implicado una jerarquización de prioridades que dejaría a las políticas de resguardo de documentación como una opción de segundo orden. En este sentido, cabe destacar la falta de un conocimiento adecuado que permitiese ordenar y clasificar la información, y que ayudase con su dispersión hacia diversos espacios. Este desorden afecta fuertemente el acceso a la información y, por ende, el rescate de la memoria del sindicalismo en dictadura.

Finalmente, un tercer factor a considerar en el desarrollo de una historiografía sobre el sindicalismo en la dictadura tiene relación con la falta de preocupación del Estado respecto al resguardo de las memorias de los movimientos sociales. En un contexto de carencia o deficiencias en la formulación de políticas archivísticas a nivel estatal -reflejo de la ausencia de peso específico de un modelo de Estado que renuncia a ejercer sus facultades o a diseñar políticas culturales para avalar el paradigma de la privatización de la función pública-, será responsabilidad de cada organización el preocuparse por tener una política archivística o por mantener unos criterios mínimos de resguardo de la documentación poseída. En ese sentido, si observamos experiencias en el extranjero, como el caso español, el Estado ha financiado vía subsidios una red de archivos de los sindicatos Comisiones Obreras y Unión General de Trabajadores (UGT), iniciativa que también representa el propio interés de la organización sindical para resguardar su memoria de lucha contra el franquismo.

Por lo tanto, los tres factores analizados dan cuenta de una difícil realidad para generar conocimiento desde las ciencias sociales sobre el movimiento sindical en dictadura y democracia. Ahora bien, esta falta de preocupación responde también a un contexto en que la categoría o concepto trabajo perdió centralidad como factor para comprender los cambios experimentados por el movimiento sindical en los últimos 40 años. Sin embargo, en los últimos años, la emergencia de nuevas organizaciones sindicales - muchas de ellas al margen de la reconstituida CUT - y la constatación de los problemas de sustentabilidad y de equidad del neoliberalismo han llevado a nuevos debates sobre los cambios en las relaciones laborales. Recuperan su vigencia categorías como trabajo y se incorporan nuevas temáticas vinculadas a fenómenos como la flexibilidad, precariedad y tercerización laboral, de tal manera que se ha producido una renovación de los estudios laborales, lo que facilita el diálogo entre las ramas de las ciencias sociales y ayuda a complejizar los análisis sobre la dictadura y su relación con los trabajadores (Gaudichaud, 2017; Campusano, Gaudichaud, Osorio, Medel y Urrutia, 2015a y 2015b).

La multiplicidad de fuentes, archivos y centros de documentación relativos al mundo del trabajo nos lleva a examinar ciertos supuestos que han sustentado las principales interpretaciones sobre la trayectoria del sindicalismo chileno desde los tiempos de la Unidad Popular. Así, como se ha mencionado en páginas anteriores, se construyó una imagen de un movimiento sindical fuerte vinculado a la izquierda que alcanzó la plenitud de su poder durante el gobierno de Salvador Allende. Sin embargo, tal interpretación choca con la evidencia de conflictos internos entre los propios partidos de izquierda y la presencia de un sindicalismo de corte demócrata cristiano, que alcanzó cuotas importantes de poder en la propia CUT, hasta llegar a ser la tercera fuerza política en las elecciones sindicales del año 1972. Por otra parte, las cifras de afiliación sindical, si bien fueron las más altas de la historia - por encima del $30 \%$ en 1973-, deben ser analizadas con cuidado, a razón del tamaño de los sindicatos y la cantidad de socios. Este análisis se puede sustentar en la revisión contenida en el fondo DIRELA del ARNAD y en los datos de afiliación sindical de la Dirección del Trabajo.

Por lo tanto, si se realiza el análisis de la evolución del sindicalismo entre la fase democrática anterior al golpe y la fase de la dictadura, el concepto de crisis puede ser reinterpretado en referencia a una situación de cambio abrupto o de conjunción de problemas, lo que permitiría justificar actitudes o visiones políticas sobre el estado del sindicalismo. Estas visiones resultan nostálgicas de un sindicalismo de corte clásico, vinculado 
a un tipo de modelo económico de carácter desarrollista y a formas de acción sindical basadas en repertorios clásicos de protestas.

El análisis de las fuentes provenientes de tiempos de la dictadura da cuenta de un movimiento sindical heterogéneo en su composición social, diverso políticamente y con distintos grados de adaptación o respuesta al Plan Laboral. En efecto, la revisión de la prensa nacional y regional muestra la existencia de un sindicalismo de corte oficialista, con importante presencia en la industria del acero, el cobre y el carbón. En este último caso, la presencia de dirigentes como José Domínguez y Miguel de Gregorio -pertenecientes al sindicato $\mathrm{n}^{\circ}$ 1 de la Empresa Nacional del Carbón (ENACAR) y elegidos con amplias mayorías- sería más llamativa aún por la tradicional relación entre el partido Comunista y los sindicatos del carbón. Es decir, los yacimientos del carbón fueron durante mucho tiempo bastiones de la izquierda, por lo que la presencia de dirigentes vinculados a la dictadura expresaría la voluntad de ésta de refundar la sociedad sobre nuevas bases para ser testigo de la aparición de un tipo de sindicalismo de carácter economicista, preocupado de asegurar los intereses económicos de sus representados por sobre cualquier demanda de carácter más global. Entonces, según el uso de las fuentes y la metodología aplicada, podrán variar las interpretaciones sobre el movimiento sindical en la dictadura: o bien se sobreestimará la presencia de los partidos políticos en el sindicalismo, o bien, por el contrario, se subestimarán los efectos del modelo neoliberal en la conducta de los trabajadores en sus relaciones con los patrones al interior de las empresas.

Esta misma emergencia de un sindicalismo de derecha se puede explicar también por la acción desarrollada por las escuelas de formación política implementadas por la secretaría de los gremios. Este organismo, dependiente de la Dirección de Organizaciones Sociales del Ministerio Secretaría General de Gobierno, era una institución en la que convivían gremialistas y nacionalistas ${ }^{10}$. Aunque es complejo poder evaluar el impacto real de estos cursos de formación, su sola presencia viene a matizar las afirmaciones respecto a la inexistencia de una política hacia los sindicatos por parte de la dictadura, más bien, existiría la voluntad de construir un sindicalismo despolitizado, proclive al entendimiento con los empresarios y el gobierno, declaraciones que se pueden observar en testimonios de dirigentes sindicales recogidos por la prensa de la época.

La lectura de las fuentes entrega nuevas posibilidades para investigar las expresiones regionales de las organizaciones sindicales nacionales, o las de carácter intermedio vinculadas a la oposición. Así, tanto a través de los boletines - publicados por sindicatos y ONG vinculadas al trabajo sindical- contenidos en el Fondo de Organizaciones Sociales del ARNAD, como del registro de actividades de las organizaciones contenido en la prensa nacional y regional, se muestran los esfuerzos de los dirigentes por conformar estructuras regionales del Comando Nacional de Trabajadores -formado en mayo de 1983-, que es la principal instancia unitaria de la oposición sindical a la dictadura (Araya, 2014). Así, en capitales regionales como Concepción y Valparaíso se formaron comandos regionales de trabajadores, los cuales agrupaban a los principales sindicatos de sus zonas, y actuaban de acuerdo con otras organizaciones sociales en las jornadas de movilización social. La tarea de aumentar la sindicalización fue un constante objetivo de las federaciones de rama, las cuales estaban despojadas de gran parte de sus atribuciones y funciones por el Plan Laboral, pero tenían la posibilidad de asesorar a los sindicatos locales en la negociación colectiva o de ayudar en la capacitación de dirigentes. Así, en la ciudad de Concepción encontramos la actividad de la Confederación del Cuero y el Calzado, la Federación del Petróleo, Federación de la Madera, Agrupación de Educadores de Chile, entre otras. Estas organizaciones, mediante la visita de sus dirigentes nacionales, conforman diversas filiales, hecho que muestra la dimensión regional del sindicalismo opositor. Esto último constituye un área insuficientemente investigada en los trabajos sobre la dictadura.

Otra posible fuente de información sobre la actividad sindical se encuentra en los archivos de las empresas públicas como la Empresa Nacional de Telecomunicaciones, la Sociedad Química de Chile o la Compañía Chilena de Electricidad, las cuales experimentaron procesos de privatización en la década de los 80 bajo la fórmula del capitalismo popular. En este sentido, los estudios sobre las privatizaciones se han referido al 
impacto económico o legal de los cambios en estas empresas, y han dejado de lado el impacto en las relaciones laborales en el interior de estas empresas, a pesar de que gran parte de los sindicatos se opusieron a las privatizaciones, como puede rastrearse en la prensa de oposición y en los boletines de los propios sindicatos.

Otro planteamiento generalizado en los estudios sobre el sindicalismo en la dictadura se refiera a la escasa combatividad de los trabajadores y la casi nula presencia de acciones reivindicativas como huelgas, mítines o tomas de fábricas. Sin embargo, al observarse la información de prensa y los boletines sindicales, estas afirmaciones generales se pueden matizar a nivel regional, pues se menciona una serie de procesos de negociación colectiva que involucran a empresas de distinto tamaño y el desarrollo de conflictos colectivos de envergadura. Entre ellos puede señalarse la huelga marítima liderada por la COMACH, organización presidida por el líder sindical demócrata cristiano Eduardo Ríos, quien a la vez era presidente de la Central Democrática de Trabajadores de Chile (CDT), vinculada a la oposición sindical conservadora. Además, al revisar la documentación de la Dirección del Trabajo se pueden encontrar las menciones tanto a conflictos derivados de los llamados a protestas en la década de los 80 , como a prácticas antisindicales de dueños de empresas, e incluso informes de los secretarios regionales sobre la actividad sindical de la oposición.

Otra problemática de reciente estudio en Chile se refiere a la complicidad económica empresarial con la dictadura cívico-militar. En efecto, a partir de testimonios de víctimas de violaciones a los derechos humanos, contenidos en los informes de la Comisión de Verdad y Reconciliación y en la documentación producida por los organismos defensores de los DD.HH. se ha podido identificar la participación de civiles en acciones represivas contra dirigentes sindicales o contra trabajadores en general. Estas situaciones en pocos casos han sido llevadas a la justicia, tal el caso del asesinato de trabajadores pertenecientes a la planta industrial de Laja perteneciente a la Compañía Manufacturera de Papeles y Cartones (Rebolledo, 2016). Entonces, a partir de la relectura de las fuentes se pueden explorar nuevas vías para analizar la violencia antisindical, elemento fundamental para comprender los cambios en las trayectorias del sindicalismo chileno en el periodo.

Una serie de fuentes de origen sindical se puede encontrar en archivos ubicados en países extranjeros, como España, que es de los países con más importantes archivos con documentación sindical chilena. Esta situación se explica por los importantes grados de colaboración entre los sindicatos españoles, el exilio y las organizaciones chilenas. De esto modo, en los archivos de los sindicatos españoles, como Comisiones Obreras y la Unión General de Trabajadores, se encuentra importante información emanada de los sindicatos chilenos, reflejo de la dispersión de las fuentes - problema mencionado anteriormente-. Estos documentos contienen datos que sirven para reconstruir en parte la solidaridad hacia la causa chilena que tuvo el Primer Mundo, cuyas expresiones fueron la entrega de recursos financieros y logísticos, y gestiones en pro de la liberación de detenidos. Además, se encuentra documentación interna producida por organizaciones como la Coordinadora Nacional Sindical, el Comando Nacional de Trabajadores (CNT) y la Central Democrática de Trabajadores. Esta información es rica en detalles referidos a las orientaciones desarrolladas por estas organizaciones para enfrentar a la dictadura.

La lectura de las fuentes entrega una visión del sindicalismo que se aleja del tradicional sello "obrerista" que habría tenido el movimiento sindical según sus críticos. De este modo, las acciones sindicales aparecen protagonizadas por sectores vinculados a las clases medias, como profesores, empleados de empresas estatales y obreros de áreas estratégicas como el cobre y el petróleo. En cambio, la mayor capacidad de movilización será ejercida por pobladores, cesantes y estudiantes, sectores que no siempre actuarán de consuno con las organizaciones sindicales como el CNT, que era uno de los principales convocantes a las jornadas de protesta. Entonces, para los estudios sobre el mundo del trabajo, el desafío será recoger los cambios en la capacidad de movilización de los trabajadores organizados, las transformaciones en la estructura productiva, societal y en la propia mentalidad de los sujetos, expuestos a fenómenos como el consumismo y el individualismo, cambios que ayudarían a encontrar explicaciones a las diversas conductas de los sindicatos ante las políticas de privatización o de capitalismo popular. 
Por lo tanto, la información recogida en las fuentes demuestra la potencialidad y desafíos de los estudios sobre la dictadura en el plano sindical. Conocer las características de un movimiento sindical más diversificado, permite comprender las relaciones entre el sindicato y el resto de la sociedad, en esferas que van más allá de lo estrictamente laboral, para así poder evaluar el grado de incidencia del sindicalismo en la sociedad chilena de las últimas décadas.

\section{Conclusiones}

A través del artículo hemos analizado la relevancia y necesidad de los estudios históricos sobre el movimiento sindical en la dictadura cívico-militar, esto es, porque si bien, se constata una escasez de investigaciones sobre el tema, muchas de ellas, dependientes de la coyuntura política en que fueron desarrolladas -imposición del Plan Laboral en 1979- y vinculadas especialmente a la sociología del trabajo, estos enfoques sin perder completa vigencia, resultan insuficientes para comprender la complejidad del periodo autoritario, de modo que la perspectiva histórica resulta fundamental para identificar los procesos de continuidad y cambio en las trayectorias del movimiento social.

En este sentido, constatamos los factores que han dificultado las investigaciones sobre el sindicalismo en dictadura, problemas vinculados al acceso de las fuentes, ya sean provenientes de instituciones estatales o de las propias organizaciones sindicales. Así, si bien está reconocido legalmente el derecho de acceso a la información, en la práctica este derecho se ve mediatizado por factores internos -disponibilidad presupuestaria, falta de coherencia política archivística estatal- y también factores externos, falta de conciencia del valor de los archivos como patrimonio inmaterial de la nación. La convergencia de estos factores genera un cuadro que contribuye a un estado de dispersión de las fuentes sobre la dictadura, a pesar del valor que tienen para reconstruir la historia reciente del país.

No obstante, a través del examen de las fuentes y la aplicación de variadas metodologías, tanto cuantitativas como cualitativas, se pueden identificar diversas tendencias u orientaciones político-sindicales, distintos tipos de organizaciones y grados de actividad sindical en el ámbito local, regional o nacional. Por lo tanto, uno de los retos de la historiografía laboral es asumir esta heterogeneidad de las expresiones sindicales o de conducta de los trabajadores, de manera de poder desarrollar una visión más amplia de los movimientos sociales durante la dictadura y el posterior periodo de transición, tributario en gran medida del primero.

Así, el análisis de las fuentes periódicas, estatales, sindicales y orales ofrecen posibilidades de renovación a una "vieja historia", puesto que muchos de los problemas que afectan a los trabajadores y trabajadoras siguen siendo semejantes a los de los pioneros del movimiento obrero a fines del siglo XIX y comienzos del siglo $\mathrm{XX}$, problemas que apuntan a la explotación indiscriminada de la fuerza de trabajo, la subordinación del trabajador al capital y la hostilidad del Estado y sus poderes fácticos ante los sindicatos. Por lo tanto, el estudio documentado y crítico de las condiciones de vida de los trabajadores y sus formas de organización y resistencia durante la dictadura cívico-militar pueden ayudar a encontrar herramientas de análisis e interpretación que permitan superar el pesado legado del autoritarismo en la sociedad chilena.

\section{REFERENCIAS}

Álvarez, R. (2012). El Plan Laboral y la negociación colectiva: Łorigen de un nuevo sindicalismo en Chile?, 1979-1985. Boletin del Instituto de Historia Argentina y Americana Emilio Ravignani, 35/36.

Álvarez, R. (2010) Represión o integración. La política sindical del régimen militar. 1973-1980. Historia, II(43).

Angell, A. (1974). Partidos políticos y movimiento obrero en Chile. México: Ediciones Era.

Aravena, A. y Núñez, D. (EdS.). (2009). El renacer de la buelga obrera en Chile. El movimiento sindical en la primera década del siglo XXI. Santiago: LOM Editores. 
Araya, R. (2012). Del combate a la dictadura a la preservación de la democracia. Movimiento sindical y politicas de concertación social. Los casos de Chile y España (Tesis para optar al grado de Doctor en Historia), Universidad Autónoma de Barcelona.

Araya, R. (2014). Cambios y continuidades en el movimiento sindical chileno en los años 80. El caso del Comando Nacional de Trabajadores. Historia, I(47).

Araya, R. (2015). Organizaciones Sindicales en Chile. De la resistencia a la política de los consensos. 1983-1994. Santiago: Ediciones Universidad Finis Terrae.

Araya, R. (2017). "Ha llegado el momento de decir basta". El movimiento sindical y la lucha por la democracia en Chile, 1973-1990. Izquierdas, 37, 191-211.

Ayala, J. (2016). Historia del movimiento sindical de Huachipato, 1970-2013. Concepción: Escaparate Ediciones.

Barrera, M, Henríquez, H. y Selame, T. (1985). Sindicatos y estado en el Chile actual. Santiago: UNRISD.

Bohoslavsky, J.P, Fernández, K. y Smart, S. (2019). Un país desigual a la fuerza. Complicidad económica con la dictadura chilena. Santiago: Lom Ediciones.

Campero, G. y Valenzuela, J.A. (1981). El movimiento sindical en el capitalismo autoritario (1973-1981). Santiago: ILET.

Campusano, K., Gaudichaud, F., Osorio, S., Medel, R. y Urrutia, M. (2017). Conflictividad laboral y politización en los procesos de trabajo. Una propuesta teórica para el análisis de la trayectoria reciente del movimiento sindical chileno. Nuevos Mundos Mundos Nuevos. Recuperado de https://journals.openedition.org/nuevomundo/70 626

Campusano, K., Gaudichaud, F., Osorio, S.; Medel, R.; y Urrutia, M. (2015). Conflictividad laboral y politización en los procesos de trabajo. Una aproximación a las orientaciones políticas del sindicalismo en el "neoliberalismo maduro", 1989-2015. Nuevos Mundos Mundos Nuevos. Recuperado de https://journals.openedition.org/nue vomundo/70626

Campero, G. (1984). Los gremios empresariales en el periodo 1970-1983: comportamiento sociopolitico y orientaciones ideológicas. Santiago: ILET.

Drake, P. (1996a). Labor Movements and Dictatorship. The Southern Cone in Comparative Perspective. Baltimore: The Johns Hpopkins University Press.

Drake. P. (1996b). El movimiento obrero en Chile. De la Unidad Popular a la Concertación. Revista de Ciencia Politica, $X X I I I(2)$.

Frank, V. (2000). El movimiento sindical en la nueva democracia chilena. Perspectivas de los dirigentes sindicales de base: ¿Hechos o ficción?. Revista Universum, (15).

Frías, P. (1988). El movimiento sindical chileno en la lucha por la democracia. Santiago: PET.

Gárate, M. (2012). La revolución capitalista de Chile (1973-2003). Santiago: Ediciones Universidad Alberto Hurtado.

Garcés, M. y Milos, P. (1988). FOCH, CTCH, CUT: las centrales unitarias en la historia del sindicalismo chileno. Santiago: ECO Comunicaciones.

Garcés, M. y De la Maza, G. (1985). La explosión de las mayorias: protesta nacional 1983-1984. Santiago: ECO Comunicaciones.

Garretón, M. (2012). Neoliberalismo corregido y progresismo limitado: los gobiernos de la concertación en Chile, 1990-2010. Santiago: Editorial Arcis.

Gaudichaud, F. (2017). Las fisuras del neoliberalismo chileno maduro chileno. Trabajo, "Democracia protegida" y conflicto de clases. Buenos Aires: CLACSO.

Grez, S. (1997). De la regeneración del pueblo a la buelga general: génesis y evolución histórica del movimiento popular en Chile (1810-1890). Santiago: DIBAM.

Jobet, J. (1951). Ensayo critico del desarrollo económico-social de Chile. Santiago: Universitaria.

Klubock, T. (2012). Class, Community, and Neoliberalism in Chile: Copper Workers and the Labor Movement during the Pinochet Dictatorship and the Restoration of Democracy. En P. Winn, Victims of the chilean miracle: workers and neoliberalism in the Pinochet era, 1973-2002 (pp. 209-260). Durham: Duke University Press. 
López, A. (2013). Desarticulación y resistencia: movimiento obrero y sindicalismo en dictadura: Chile y Argentina, 1973-1983 (Tesis de Magister Inédita), Facultad de Filosofía y Humanidades Universidad de Chile, Santiago.

Ortiz Letelier, F. (1956). El movimiento obrero en Chile (1891-1919). Madrid: Michay.

Osorio, S. (2015). Trayectoria y cambios en la politica del movimiento sindical en Chile, 1990-2010: el caso de la CUT, entre la independencia politica y la integración al Bloque Histórico Neoliberal (Tesis de Magister Inédita), Facultad de Humanidades, Universidad de Santiago de Chile, Santiago .

Pinto, J. (1998). Trabajos y rebeldias en la pampa salitrera: el ciclo del salitre y la reconfiguración de las identidades populares (1850-1900). Santiago: Ediciones Universidad de Santiago.

Pizarro, C. (1985). La huelga obrera en Chile: 1890-1970. Santiago: SUR Ediciones.

Ponce, C., Santibáñez, C. y Pinto J. (comps.). (2017). Trabajadores y Trabajadoras. Procesos y acción sindical en el neoliberalismo chileno 1979-2017. Valparaíso: América en Movimiento.

Ramírez Necochea, H. (1956). Historia del movimiento obrero en Chile: antecedentes siglo XIX. Santiago: Austral.

Rebolledo, J. (2016). A la sombra de los cuervos. Los cómplices civiles de la dictadura. Santiago: Planeta.

Rojas Flores, J. (2000). Los trabajadores en la historiografía chilena: balance y proyecciones. Revista de Economía y Trabajo, 2 vol. (10), Santiago: PET .

Ruiz-Tagle, J. (1985). El sindicalismo chileno después del plan laboral. Santiago: PET.

Ruiz, C. (2015). De nuevo la sociedad. Santiago: LOM Ediciones.

Salazar, G. (1985). Labradores, peones y proletarios: formación y crisis de la sociedad popular chilena del siglo XIX. Santiago: SUR Ediciones.

Salazar, G. y Pinto, J. (1999). Historia Contemporánea de Chile, (vols. 1-5). Santiago: LOM Ediciones.

Samaniego, A. (2016). Unidad sindical desde la base. La Central Única de Trabajadores, Chile 1953-1973. Santiago: Ariadna Ediciones.

Stillerman, J. (2005). Continuidades, rupturas y coyunturas en la transformación de los obreros de MADECO, SA. Revista Politica (44).

Tinsmann, H. (2016). Se compraron el modelo. Consumo, uva y la dinámica transnacional: Estados Unidos y Chile durante la Guerra Fría. Santiago: Ediciones Universidad Alberto Hurtado.

Vergara, A. (2018). Writing about Workers, Reflecting on Dictatorship and Neoliberalism: Chilean Labor History and the Pinochet Dictatorship. International Labor and Working-Class History, (93), Spring .

Winn, P. (2012). Victims of the chilean miracle: workers and neoliberalism in the Pinochet era, 1973-2002. Durham: Duke University Press.

Zapata, F. (2010). La huelga de los mineros de La Escondida de Agosto de 2006. Si Somos Americanos. Revista de Estudios Transfronterizos, Vol. X, (2).

\section{Notas}

1 Entre los años 2006 a 2008 se experimentó un proceso de revigorización del movimiento sindical, encarnado fundamentalmente en la acción de los trabajadores subcontratistas de las áreas más dinámicas de la economía.

2 Un completo balance de la historiografía marxista se puede revisar en Jorge Rojas Flores (2000), Los trabajadores en la historiografía chilena: balance y proyecciones, Revista de Economía y Trabajo, PET.

3 DERTO, Estadísticas sindicales, Santiago de Chile, 1974.

4 José Isla (1979). Estadísticas sindicales chilenas 1970-1977, Santiago: Facultad de Ciencias Económicas y Administrativas Universidad de Chile.

5 Algunas versiones de los capítulos del libro de Ruiz-Tagle se pueden encontrar en la revista Mensaje, perteneciente a la Congregación de la Compañía de Jesús.

6 De acuerdo al Decreto Ley 5200 del año 1927, todas las instituciones públicas tienen la obligación de transferir su documentación al Archivo Nacional, dependiendo los plazos del tipo de institución, correspondiendo a 5 años el tiempo que tienen los organismos centrales para transferir su documentación.

7 La página web es www.vicariadelasolidaridad.cl 
8 La página de FASIC es http://fasic.cl/wp/archivo-documental/

9 La página de Londres 38 es http://www.londres38.cl. La página del Centro de Documentación del Museo de la Memoria es http://ww3.museodelamemoria.cl/centro-de-documentacion/

10 Una parte de la documentación del Ministerio Secretario General de Gobierno se encuentra en la biblioteca de la Participación Ciudadana, heredera del Centro de Documentación del Ministerio, el cual cambió de nombre y ubicación por efectos del terremoto del año 2010. 